\title{
Sensitivity Enhancement of Optical Receivers by Impulsive Coding
}

\author{
Peter J. Winzer and András Kalmár
}

\begin{abstract}
A theory for the signal-to-noise ratio (SNR) of optical direct-detection receivers employing return-to-zero (RZ) coding (and possibly optical preamplification) is developed. The results are valid for both signal-independent noise limited and signaldependent noise limited receivers, as well as for arbitrary optical pulse shapes and receive filter characteristics. Even if the same receiver bandwidth is used, $\mathrm{RZ}$ coding is seen to perform better than nonreturn-to-zero (NRZ) coding. Asymptotic expressions for the SNR in case of very high and very low receiver bandwidths show that the full sensitivity enhancement potential of RZ coding is exhausted at fairly moderate duty cycles. A realistic example taking into account intersymbol interference (ISI) shows that a receiver sensitivity gain (compared to $\mathrm{NRZ}$ coding) of, e.g., 3.2 $\mathrm{dB}$ can be obtained in a signal-independent noise limited receiver with a bandwidth of $80 \%$ of the data rate, using a duty cycle of three.
\end{abstract}

Index Terms - Amplified spontaneous emission (ASE), noise, nonreturn-to-zero (NRZ), ON-OFF keying, optical amplifier, receiver sensitivity, return-to-zero (RZ).

\section{INTRODUCTION}

$\mathbf{I}$ T was found by Personick in 1973 [1], and has recently been pointed out again [2] that-for a given average optical power at the receiver-return-to-zero (RZ) coding where the optical pulses occupy only a fraction of the bit duration, yields improved sensitivity over nonreturn-to-zero (NRZ) coding, where the optical pulse duration equals the bit duration. This gain is obtained even if the same receiver hardware is used. Experiments fostering this finding have also been reported recently [2]-[4]. However, the theory presented in [1] constrains the receiver output to a raised-cosine shape, and both the theory and the qualitative explanation of [2] are based, again, on a specific receiver structure (which is basically a transimpedance amplifier with a first-order low-pass characteristic). Further, [2] only considers the case where signal-independent noise (e.g., thermal noise) dominates signal-dependent noise (e.g., shot noise).

In this paper, we give a general theory of the sensitivity improvement obtained by impulsive coding, valid for arbitrary optical pulse shapes and arbitrary receive filter characteristics. We calculate the (different!) sensitivity improvements obtainable for receivers whose performance is limited by signal-independent noise and for receivers whose perfor-

Manuscript received May 7, 1998; revised September 29, 1998.

The authors are with the Institut für Nachrichtentechnik und Hochfrequenztechnik, Technische Universität Wien, Vienna A-1040 Austria (e-mail pwinzer@nt.tuwien.ac.at).

Publisher Item Identifier S 0733-8724(99)01430-9. mance is limited by signal-dependent noise. Conventional optical direct detection receivers are-apart from some designs using avalanche photodiodes - usually dominated by signalindependent noise. The same is true for some optically preamplified direct detection systems, where the signal-independent amplified spontaneous emission self-beat noise (ASE-ASE beat noise) constitutes the dominant noise source. ${ }^{1}$ Usually, however, the signal-dependent signal-ASE beat noise $(s$-ASE beat noise) determines the signal-to-noise ratio (SNR) in optically preamplified receivers. Furthermore, we address the question of how narrow the optical RZ pulses have to be to arrive at a significant sensitivity improvement and show that, unlike invoked by [2], fairly moderate duty cycles are sufficient to fully exhaust the potential of sensitivity enhancement for a given receiver bandwidth. By-products of our calculations are the optimum receiver bandwidth for given optical pulse shape and given type of electrical filter as well as general expressions for the (partly nonstationary) optical amplifier noise.

Section II of this paper, together with the appendix, provides general expressions for the signal and noise in an optical direct detection receiver. In Section III, we calculate the asymptotic behavior of the SNR for very large and very small electrical bandwidths. In a simple example in Section IV the sensitivity improvement of impulsive coding is evaluated analytically as a function of receiver bandwidth and RZ duty cycle. In Section V, we include both intersymbol-interference (ISI) and a realistic optical pulse shape and receive filter characteristic.

Throughout our work, bold print indicates stochastic processes (e.g., $\mathrm{x}(t)$ ), whose ensemble average is denoted $\langle\mathrm{x}(t)\rangle$, and whose variance reads $\sigma_{\mathbf{x}}^{2}(t)$.

\section{Signal CURRENT AND NOISE FOR ARBITRARY RECEIVER BANDWIDTHS}

We first have to clearly define the signal current and the noise terms in optical receivers. As we are interested in arbitrary ratios of the electrical receiver bandwidth to the bandwidth of the optical pulse, general expressions for signaldependent noise-involving convolutions-have to be used: It can be shown (see, e.g., [5] and [6]) that the ensemble average and the (signal-dependent) shot noise variance of the photocurrent $\mathbf{i}(t)$ produced by a deterministic optical field with

\footnotetext{
${ }^{1}$ This is the case if the bandwidth of the optical filter following the optical amplifier is much larger than the data rate.
} 
power $p(t)$ generally read $^{2}$

$$
\langle\mathbf{i}(t)\rangle=S(p * h)(t)
$$

and

$$
\sigma_{\mathbf{i}, \text { dep }}^{2}(t)=S e\left(p * h^{2}\right)(t)
$$

where $S=\eta e / h f$ denotes the detector's sensitivity ( $\eta$ is the detector's quantum efficiency, $e$ stands for the elementary charge and $h f$ represents the energy of one photon), and $h(t)$ is the impulse response of the electrical filter, normalized such that the integral over $h(t)$ is unity. ${ }^{3}$ Note from (2) that shot noise is nonstationary in general. For the case of a receiver employing an optical preamplifier with subsequent optical filtering, it is shown in the appendix that the signal-dependent $s$-ASE beat noise is of the same form as (2) and that the ASEASE beat noise is additive and stationary. The latter can thus be treated analogous to other additive noise sources (such as, e.g., thermal noise), leading to a signal-independent variance of the form

$$
\sigma_{\mathbf{i}, \text { indep }}^{2}=N_{0} B_{h}
$$

where $N_{0}$ is the (single-sided) power spectral density of the noise, assumed independent of the receiver bandwidth ${ }^{4} ; B_{h}$ is the power equivalent bandwidth of $h(t)$, defined as ${ }^{5}$ [6]

$$
B_{h}=\int_{0}^{\infty}|H(f)|^{2} d f .
$$

The function $H(f)$ denotes the Fourier transform of $h(t)$

$$
H(f)=\int_{-\infty}^{\infty} h(t) \exp (-j 2 \pi f t) d t .
$$

We define the SNR as

$$
\mathrm{SNR}=\frac{\left\langle\mathbf{i}_{1}\right\rangle^{2} F^{2}}{\sigma_{\mathbf{i}_{1}}^{2}}
$$

where $\mathbf{i}_{1}$ is the photocurrent for a mark at the optimum sampling instant (which is the one that maximizes the SNR). The parameter $F \epsilon[0,1]$ takes account of both finite extinction ratios and ISI: The bit error probability (BEP) is uniquely (see, e.g., [9]) related to the $Q$-factor, which can be expressed as

$$
Q=\frac{\left\langle\mathbf{i}_{1}\right\rangle\left(1-\frac{\left\langle\mathbf{i}_{0}\right\rangle}{\left\langle\mathbf{i}_{1}\right\rangle}\right) F_{\text {ISI }}}{\sigma_{\mathbf{i}_{1}}+\sigma_{\mathbf{i}_{0}}}
$$

${ }^{2}$ The symbol * denotes a convolution,

$$
(x * y)(t)=\int_{-\infty}^{\infty} x(\tau) y(t-\tau) d \tau .
$$

\footnotetext{
${ }^{3}$ Strictly speaking, $h(t)$ stands for the normalized convolution of the detector's impulse response with that of the electrical filter; however, since in most applications the detector bandwidth exceeds that of the electrical filter, $h(t)$ is determined primarily by the electrical filter.

${ }^{4}$ This assumption is correct if $N_{0}$ denotes the dominating ASE-ASE beat noise density in optically preamplified receivers, but has to be modified for receivers where the noise of the electronic circuitry dominates. For this case, some additional factors describing the dependence of $N_{0}$ on $B_{h}$ would have to be introduced, [7], [8], leading to worse results than predicted by our equations.

${ }^{5}$ The usual normalization to the maximum of $|H(f)|$ can be omitted in our case, as we assume that $|H(f)|$ is peaked at $f=0$, where it is unity due to the normalization of $h(t)$.
}

where $\mathbf{i}_{\mathbf{0}}$ is the photocurrent for a space at the optimum sampling instant. The term in parentheses represents the influence of a finite extinction ratio, and $F_{\text {ISI }}$ accounts for the additional eye closure due to ISI. These two terms' product equals our factor $F$ in (6). If signal-independent noise dominates $\left(\sigma_{\mathbf{i}_{1}}=\sigma_{\mathbf{i}_{0}}\right)$, the $Q$-factor becomes $\sqrt{\mathrm{SNR}} / 2$, while it is $\sqrt{\mathrm{SNR}}$ if the signal-dependent noise of the mark dominates $\left(\sigma_{\mathbf{i}_{1}} \gg \sigma_{\mathbf{i}_{0}}\right){ }^{6}$ Since it is the square of the optical power that is proportional to the electrical power, a SNR gain $G$ corresponds to a receiver sensitivity improvement of $\sqrt{G}$ in the signal-independent noise limit. In the signal-dependent noise limit, on the other hand, a SNR gain $G$ also means a receiver sensitivity improvement of $G$; this is due to the proportionality of the (electrical) noise variance and the optical power.

\section{ASYMPTOTIC EXPRESSIONS FOR THE SNR}

For the calculation of the limiting behavior of the SNR for very small and very large receiver bandwidths, consider the setup shown in Fig. 1: A single optical pulse $p(t)$, which may also have passed through an optical preamplifier with subsequent (optical) bandpass filtering limiting the ASE power, impinges on a photodetector. Let the overall optical pulse energy be $E$, regardless of the pulse duration. (We thus compare systems with the same average optical power at the receiver. While this constraint poses no problem in free-space applications, it has to be watched in fiber-based systems, where the increased peak power may induce nonlinear effects.) The electronics following the photodetector are decomposed into a frequency independent amplifier and a filter with impulse response $h(t)$. The signal-independent power spectral density of the noise current behind the amplifier is $N_{0}$; it comprises all signal independent noise sources, such as amplifier noise or ASE-ASE beat noise. We are looking for the SNR at the output of the filter. For the calculation of the SNR's asymptotic behavior, we neglect the influence of finite extinction ratio and ISI and hence set $F=1$.

\section{A. Signal-Independent Noise}

If signal-independent noise dominates the other noise sources, the SNR at the optimum sampling instant follows from (6), inserting (1) and (3), as:

$$
\mathrm{SNR}=\max _{t}\left\{\frac{S^{2}[(p * h)(t)]^{2}}{N_{0} B_{h}}\right\}
$$

where the maximum is taken over $t$. Employing the (inverse) Fourier transform, this expression can be written as

$$
\mathrm{SNR}=\max _{t}\left\{\frac{S^{2}\left[\int_{-\infty}^{\infty} P(f) H(f) \exp (j 2 \pi f t) d f\right]^{2}}{N_{0} B_{h}}\right\}
$$

where $P(f)$ stands for the Fourier transform of $p(t)$.

\footnotetext{
${ }^{6}$ Mixed cases, where none of the noise sources clearly dominates, are not considered here. They can, however, be treated with the methods presented in this paper.
} 


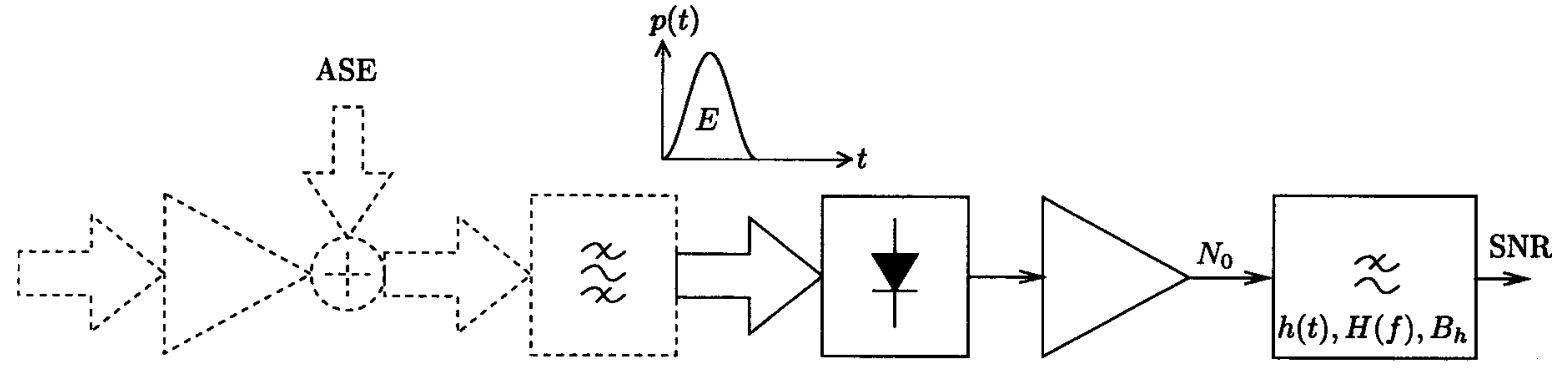

Fig. 1. Block diagram of the receiver. A single optical pulse $p(t)$, which may also have passed through an optical preamplifier with subsequent (optical) bandpass filtering, impinges on a photodetector. The overall optical pulse energy is $E$, regardless of the pulse duration. The electronics following the photodetector are decomposed into a frequency independent amplifier and a filter with impulse response $h(t)$. The signal-independent power spectral density of the noise current behind the amplifier is $N_{0}$.

1) Small Receiver Bandwidth: If the electrical bandwidth $B_{h}$ is much smaller than that of the optical pulse, we can approximate $P(f)$ by $P(0)$ in (9) and arrive at

$$
\mathrm{SNR}=\frac{S^{2} P^{2}(0)}{N_{0} B_{h}} \max _{t}\left\{\int_{-\infty}^{\infty} H(f) \exp (j 2 \pi f t) d f\right\}^{2}
$$

The integral is readily identified as $h(t) ; P(0)$ always equals $E$. We, therefore, arrive at

$$
\mathrm{SNR}=\frac{S^{2} E^{2} R}{N_{0}} \alpha_{h} b_{h}
$$

where the factor $\alpha_{h}$ is a (bandwidth-independent) property of the electrical filter

$$
\alpha_{h}=\max _{t}\{h(t)\}^{2} / B_{h}^{2}
$$

and $b_{h}=B_{h} / R$ is the receiver bandwidth normalized to the bit rate $R$. In the limit of small electrical bandwidths, the SNR is thus found to increase linearly with $b_{h}$ at a rate determined solely by the electrical circuitry (and not by the optical pulse shape). To provide some examples, $\alpha_{h}$ equals 4 , 9 , and 7.32 for rectangular, symmetrically triangular, and fifth-order Bessel-filter impulse responses $h(t)$, respectively.

2) Large Receiver Bandwidth: For electrical bandwidths large compared to the bandwidth of the optical pulse, (9) reduces to

$$
\mathrm{SNR}=\frac{S^{2}}{N_{0} B_{h}} \max _{t}\left\{\int_{-\infty}^{\infty} P(f) \exp (j 2 \pi f t) d f\right\}^{2}
$$

where we used $H(0)=1$, brought by the normalization of $h(t)$ to unit area. Identifying the integral as $p(t)$ we arrive at

$$
\mathrm{SNR}=\frac{S^{2} E^{2} R}{N_{0}} \cdot \frac{D^{2}}{b_{h}}
$$

where

$$
D=T_{b} / T_{p}
$$

is the RZ duty cycle; $T_{b}=1 / R$ is the bit duration and $T_{p}$ is the optical pulse duration defined as

$$
T_{p}=\frac{\int_{-\infty}^{\infty} p(t) d t}{\max _{t}\{p(t)\}}=\frac{E}{\max _{t}\{p(t)\}} .
$$

As expected, in the limit of large electrical bandwidths, the SNR decreases inversely proportionally to $b_{h}$.

\section{B. Signal-Dependent Noise}

If signal-dependent noise dominates, the SNR reads

$$
\mathrm{SNR}=\max _{t}\left\{\frac{S^{2}[(p * h)(t)]^{2}}{S e\left(p * h^{2}\right)(t)}\right\}
$$

where use was made of (1), (2), and (6). In the Fourier domain, this equation reads

$$
\mathrm{SNR}=\max _{t}\left\{\frac{S^{2}\left[\int_{-\infty}^{\infty} P(f) H(f) \exp (j 2 \pi f t) d f\right]^{2}}{S e \int_{-\infty}^{\infty} P(f) \tilde{H}(f) \exp (j 2 \pi f t) d f}\right\}
$$

where $\tilde{H}(f)$ is the Fourier transform of $h^{2}(t)$ (and thus the convolution of $H(f)$ with itself); it can easily be shown that $\tilde{H}(0)=2 B_{h}$.

1) Small Receiver Bandwidth: For electrical bandwidths small compared to the bandwidth of the optical pulse, (18) can be simplified to

$$
\mathrm{SNR}=\frac{S}{e} \max _{t}\left\{\frac{[P(0) h(t)]^{2}}{P(0) h^{2}(t)}\right\}
$$

and further to

$$
\mathrm{SNR}=\frac{S E}{e} .
$$

For dominating signal-dependent noise and small electrical bandwidths, the SNR is thus independent ${ }^{7}$ of $b_{h}$, regardless of the optical pulse shape and the electrical filter characteristics.

2) Large Receiver Bandwidth: If $B_{h}$ significantly exceeds the bandwidth of the optical pulse, (18) simplifies to

$$
\mathrm{SNR}=\frac{S}{2 e B_{h}} \max _{t}\left\{\frac{\left[\int_{-\infty}^{\infty} P(f) \exp (j 2 \pi f t) d f\right]^{2}}{\int_{-\infty}^{\infty} P(f) \exp (j 2 \pi f t) d f}\right\}
$$

as $H(0)=1$ and $\tilde{H}(0)=2 B_{h}$, and further to

$$
\mathrm{SNR}=\frac{S \max _{t}\{p(t)\}}{2 e B_{h}}
$$

which closely resembles the well-known expression for the shot noise limited SNR. ${ }^{8}$

\footnotetext{
${ }^{7}$ Obviously, this asymptote is only practically relevant down to receiver bandwidths at which the effect of ISI becomes significant.

${ }^{8}$ The subtle difference to the commonly used shot noise formula is the presence of the maximum of $p(t)$ instead of its time average; for a detailed discussion of this difference see [10].
} 


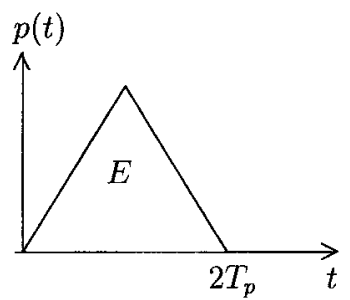

(a)

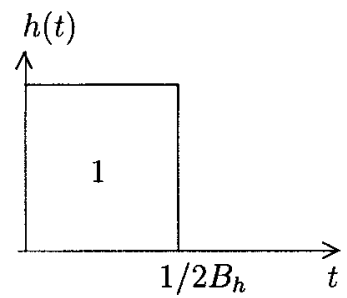

(b)
Fig. 2. (a) Optical input pulse and (b) filter impulse response used in Section IV.

\section{Analytical Solutions for a Simple Example}

We now demonstrate the principle of sensitivity enhancement by impulsive coding using a simple example, for which analytical solutions for the SNR as a function of $b_{h}$ can be found. This example does not take account of real-world pulse shapes and ISI; the analysis of a representative system including ISI is postponed to the next section.

Consider a single, symmetrically triangular optical pulse $p(t)$ of energy $E$ incident on a receiver with rectangular impulse response $h(t)$; the two functions are shown in Fig. 2. Using (1)-(3), the SNR (6) can readily be calculated. The results are

$$
\mathrm{SNR}_{\text {indep }}= \begin{cases}\frac{S^{2} E^{2} R}{N_{0}} 4 b_{h} & b_{h}<D / 4 \\ \frac{S^{2} E^{2} R}{N_{0}} \frac{D^{2}}{b_{h}}\left(1-\frac{D}{8 b_{h}}\right)^{2} & b_{h}>D / 4\end{cases}
$$

for the signal-independent case, and

$$
\mathrm{SNR}_{\mathrm{dep}}= \begin{cases}\frac{S E}{e} & b_{h}<D / 4 \\ \frac{S E}{e} \frac{D}{2 b_{h}}\left(1-\frac{D}{8 b_{h}}\right) & b_{h}>D / 4\end{cases}
$$

for the signal-dependent case. The results (23) and (24) are shown in Fig. 3 as solid lines. The dashed curves represent the asymptotic expressions (11), (14), (20), and (22). Two sets of curves are presented, corresponding to $D=1$ (which can be associated with NRZ) and $D=2$ (which is RZ with duty cycle 2).

For the signal-independent case, Fig. 3(a), we find that the choice of a receiver of normalized bandwidth $b_{h}=0.4$, which is near the optimum in this case, ${ }^{9}$ leads to a SNR gain $G$ of $1.3 \mathrm{~dB}$-corresponding to a receiver sensitivity gain of $0.65 \mathrm{~dB}$-if we use RZ with duty cycle 2 instead of NRZ. Note that no additional gain can be obtained for higher duty cycles at this specific receiver bandwidth without changing the electrical filter characteristics! (As pointed out in Section IIIA1, modifying the electrical filter characteristics changes the slope of the low-bandwidth asymptote and thus permits higher limiting values for the SNR.)

For the signal-dependent case, Fig. 3(b), RZ with duty cycle 2 is seen to exhaust the full potential of sensitivity enhancement at a normalized receiver bandwidth of 0.4 by far, yielding a SNR (as well as receiver sensitivity) gain $G$ of $0.7 \mathrm{~dB}$. Note that, while in the signal-independent case

\footnotetext{
${ }^{9}$ It will be seen in the next section that the choice of more realistic waveforms together with the influence of ISI shifts the optimum receiver bandwidth to higher, more common values of about 0.6.
}

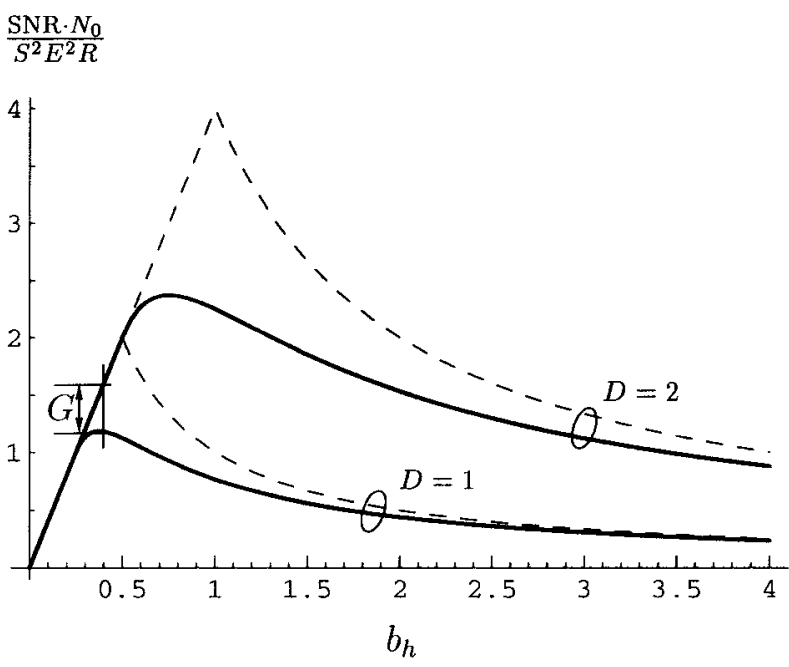

(a)

$$
\frac{\mathrm{SNR} \cdot e}{S \cdot E}
$$

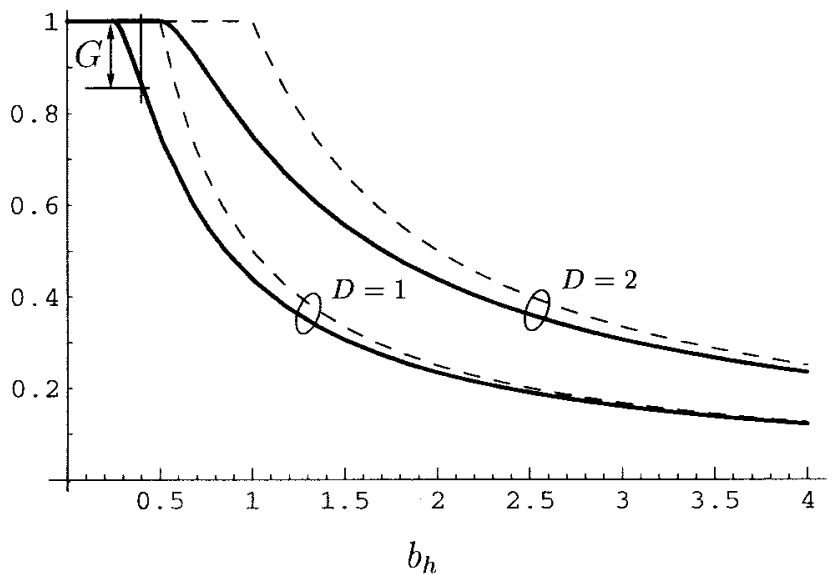

(b)

Fig. 3. Normalized SNR for the pulse shapes depicted in Fig. 2 and for either dominating signal-independent noise (a) or dominating signal-dependent noise (b). The solid lines represent (23) and (24), while the dashed lines show the asymptotic expressions (11), (14), (20), and (22). Two sets of curves corresponding to RZ duty cycles $D=1$ and $D=2$ are shown. Also indicated is the maximum SNR gain $G$ at $b_{h}=0.4$.

arbitrary ${ }^{10}$ high sensitivity improvements can be obtained by increasing the duty cycle and increasing $b_{h}$, no further gain can be achieved in the signal-dependent case.

\section{ANALYSIS OF A REPRESENTATIVE SySTEM INCLUDING ISI}

We now discuss the achievable sensitivity gain of a realistic optical direct detection receiver taking into account both a representative optical pulse form and electrical filter characteristic as well as ISI.

For the optical pulses $p(t)$ we choose the raised cosine dependence

$$
p(t)= \begin{cases}\frac{E}{T_{p}} \sin ^{2}\left(\frac{t \pi}{2 T_{p}}\right), & 0<t<2 T_{p} \\ 0, & \text { elsewhere }\end{cases}
$$

\footnotetext{
${ }^{10}$ As pointed out above, this is only the case if $N_{0}$ does not increase with
} 


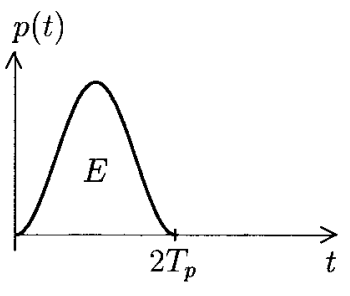

(a)

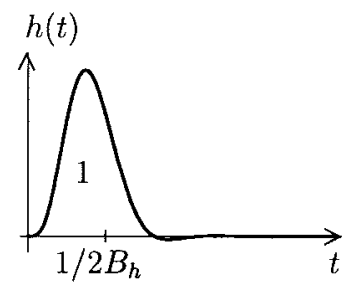

(b)
Fig. 4. (a) Optical input pulse and (b) filter impulse response used in Section V.

and assume a fifth-order Bessel-filter characteristic for the receiver, whose Laplace transform $H(s)$ is given by

$$
H(s)=\frac{945}{s^{5}+15 s^{4}+105 s^{3}+420 s^{2}+945 s+945} .
$$

The two pulse shapes $p(t)$ and $h(t)$ are shown in Fig. 4(a) and (b), respectively. We now include the influence of ISI, as mentioned along with (6), by the factor $F \epsilon[0,1]$. Obviously, $F$ is bit pattern dependent. Worst ISI, i.e., minimum $F$, is obtained at the highest eye-closure. We thus compare a "...0001000 ..." pattern (which is the ISI-free, single-pulse case discussed so far) with the worst case, a "...1110111 ..." pattern $^{11}$, and arrive at the general expression for $F$

$$
F=\frac{\max _{t}\left\{(p * h)(t)-\sum_{k=-N, k \neq 0}^{N}(p * h)\left(t-k T_{b}\right)\right\}}{(p * h)\left(t_{\max }\right)}
$$

where $t_{\max }$ is the optimum sampling instant and $2 N$ is the number of pulses that significantly contribute to the eyeclosure.

Numerically evaluating expression (6) for the SNR leads to the curves shown in Fig. 5(a) for the signal-indepenent case and Fig. 5(b) for the signal-depenent case. The plots show the asymptotes found in Section III (dashed) together with the numerically obtained curves for three different duty cycles $D$ (solid). The curves neglecting ISI approach the asymptotes for $b_{h} \rightarrow 0$, whereas the curves with worst ISI approach zero SNR at $b_{h} \approx 0.2$. The true (bit pattern dependent) curves lie all within the area between these two extremes; for the sake of clearness, this area is hatched for $D=1$.

For the signal-independent case and $D=1$, which represents NRZ coding, we find that the optimum receiver bandwidth is about 0.6 times the data rate; this agrees with general knowledge. Using RZ with $D=2$ and the same receiver bandwidth, a sensitivity improvement from 1.7 to $2.3 \mathrm{~dB}$ (a SNR gain from 3.4 to $4.6 \mathrm{~dB}$ ) can be achieved compared to NRZ. At $D=3$, the sensitivity gain from 2.2 to $2.8 \mathrm{~dB}$ is already very close to the optimum gain from 2.8 to 3.2 $\mathrm{dB}$ achievable at this bandwidth. Thus, impulsive coding with moderate duty cycles yields high sensitivity enhancements and the limiting gain is reached at fairly low duty cycles. Higher gains can be achieved if one is willing to increase the receiver bandwidth, a well-known fact in communications engineering.

\footnotetext{
${ }^{11}$ It may happen for some configurations (where $h(t)$ becomes significantly negative) that, at specific receiver bandwidths, other bit patterns yield worst ISI; for these cases, the factor $F$ defined in (27) is rather an approximation for the worst ISI than its upper bound.
}

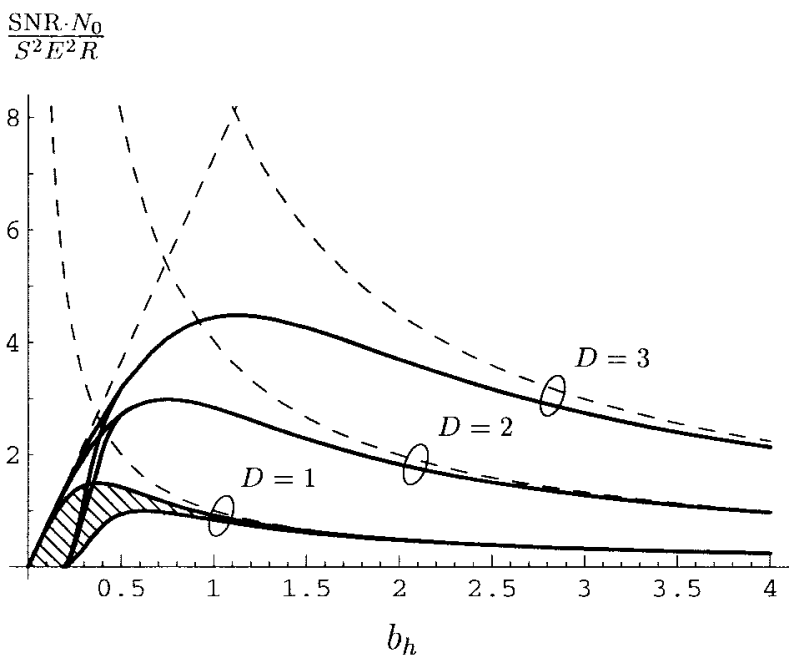

(a)

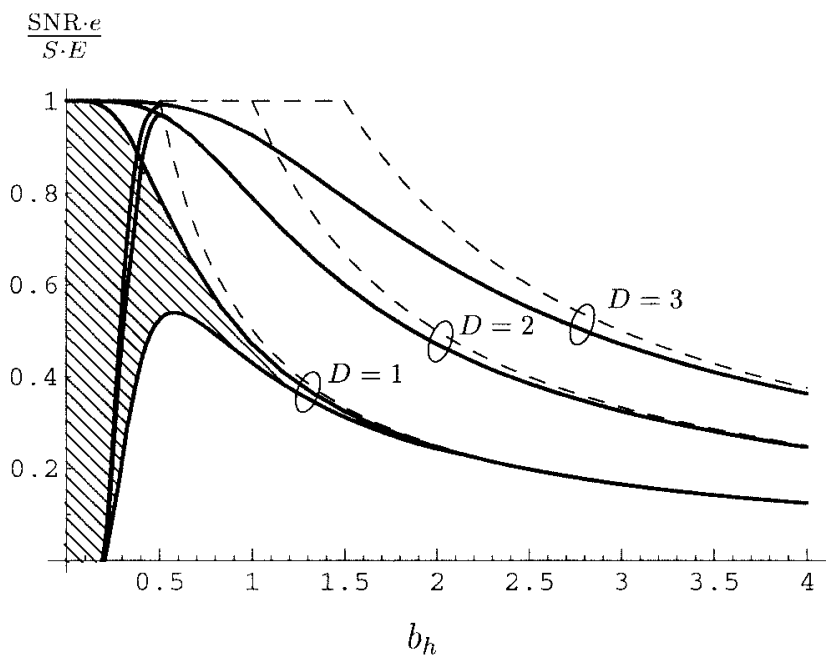

(b)

Fig. 5. (a) Normalized SNR calculated using the waveforms given in Fig. 4 for dominating signal-independent noise and (b) dominating signal-dependent noise. The solid lines represent (6), while the dashed lines show the asymptotic expressions (11), (14), (20), and (22). Three sets of curves corresponding to duty cycles $D=1,2,3$ are shown.

For the signal-dependent case [Fig. 5(b)], the receiver sensitivity enhancement at $b_{h}=0.6$ lies between $1.3 \mathrm{~dB}$ and $2.4 \mathrm{~dB}$ for RZ with $D=2$, and between 1.5 and $2.6 \mathrm{~dB}$ for $D=3$. The optimum sensitivity gain is from 1.6 to $2.7 \mathrm{~dB}$ but, in contrast to the signal-independent case, with no further improvement potential.

\section{CONCLUSION}

Using impulsive coding at the transmitter, the sensitivity of optical direct detection receivers (including those with optical preamplification) can be enhanced by several $\mathrm{dB}$ compared to NRZ coding for the same average power at the receiver. This is true even if the same receiver hardware is employed. We presented a theory of the achievable sensitivity gain, valid for the equally important classes of signal-independent noise limited and signal-dependent noise limited receivers, as 
well as for arbitrary optical pulse shapes and receive filter characteristics.

We arrived at asymptotic expressions for the SNR for very low and very high electrical receiver bandwidths and pointed out that the low-bandwidth asymptotes represent the ultimate sensitivity obtainable by impulsive coding. These asymptotes are reached at fairly moderate RZ duty cycles of about three for a technically representative system. The generation of extremely narrow optical pulses at the transmitter is thus not required.

In signal-dependent noise limited receivers, the maximum sensitivity gain is limited to about $2 \mathrm{~dB}$, showing no further improvement possibilities by increasing the receiver bandwidth. The performance of signal-independent noise limited receivers, on the other hand, can be improved by about $3 \mathrm{~dB}$ at a receiver bandwidth of about 0.6 times the data rate, with additional potential if the receiver bandwidth is increased (and if the signal-independent noise power spectral density is not significantly increased, too).

Our calculated sensitivity gain at a bandwidth of 0.8 times the data rates and an RZ duty cylce of three is about 3.2 $\mathrm{dB}$, with a full (asymptotic) potential of about $3.8 \mathrm{~dB}$. These values agree well with the results of [3] where $3.5 \mathrm{~dB}$ were measured in a $10-\mathrm{Gb} / \mathrm{s}$ optically preamplified direct-detection system with a duty cycle of slightly more than three.

\section{APPENDIX}

\section{OPTICAL AMPLIFIER NOISE CHARACTERISTICS}

Optical amplifier noise has been widely discussed on a photon counting basis (see, e.g., [11] and the references cited therein). However, when studying optoelectronic systems, we are rather interested in photocurrent statistics, which cannot be generally related to photon counting statistics, as shown in [12]. Apart from the quantum-mechanically correct treatment based on photon counting, there are also semiclassical derivations (see, e.g., [13]) that assume the spontaneous emission noise to be an additive, stationary, and circularly symmetric Gaussian process; the semiclassical treatment yields the correct results for classical fields at the amplifier input. Furthermore, it seems to be the only approach capable of incorporating the impulse response of the detector and the electronics into the theory for the detection of optically amplified radiation.

Astonishingly, all the semiclassical treatments presented so far assume the electronics' bandwidth to exceed that of the optical signal at some point within the derivations, and thus do not account for arbitrary filter impulse responses. We therefore present in this appendix a general semiclassical derivation of the (photocurrent) noise associated with the detection of optically amplified radiation that fully takes account of the detector's and the electronics' impulse responses.

Assume that the normalized, ${ }^{12}$ complex optical field after the optical bandpass following the optical amplifier reads:

$$
\mathbf{V}(t)=V_{s}(t)+\mathbf{V}_{\mathbf{n}}(t)
$$

\footnotetext{
${ }^{12}$ All field quantities are normalized so that their squared magnitude represents the optical power, i.e. the field's intensity integrated over the detector area.
}

where $V_{s}(t)$ represents the complex envelope of the deterministic, amplified optical signal and $\mathbf{V}_{\mathbf{n}}(t)$ stands for the amplified spontaneous emission (ASE) noise, which is assumed to be a stationary, circularly symmetric, complex Gaussian process. It is shown in [5] that ensemble average and variance of the photocurrent produced by the detector under illumination with $\mathbf{V}(t)$ read

$$
\langle\mathbf{i}(t)\rangle=S\left(\left\langle|\mathbf{V}|^{2}\right\rangle * h\right)(t)
$$

and

$$
\begin{aligned}
\sigma_{\mathbf{i}}^{2}(t)= & S e\left(\left\langle|\mathbf{V}|^{2}\right\rangle * h^{2}\right)(t) \\
& +S^{2} \int_{-\infty}^{\infty} \int_{|\mathbf{V}|^{2}}(\tau, \tilde{\tau}) h(t-\tau) h(t-\tilde{\tau}) d \tau d \tilde{\tau}
\end{aligned}
$$

where $C_{|\mathbf{V}|^{2}}(\tau, \tilde{\tau})=\left\langle|\mathbf{V}(\tau)|^{2}|\mathbf{V}(\tilde{\tau})|^{2}\right\rangle-\left\langle|\mathbf{V}(\tau)|^{2}\right\rangle\left\langle|\mathbf{V}(\tilde{\tau})|^{2}\right\rangle$ is the autocovariance function of $|\mathbf{V}(t)|^{2}$. Simplification of the ensemble average (29) is straightforward and yields

$$
\langle\mathbf{i}(t)\rangle=S(p * h)(t)+S P_{n}
$$

where use was made of the abbreviations $p(t)=\left|V_{s}(t)\right|^{2}$ for the (amplified) signal power, and $P_{n}=\left\langle\left|\mathbf{V}_{\mathbf{n}}(t)\right|^{2}\right\rangle$ for the average ASE power after filtering with the optical bandwidth $B_{0}$. Additionally, use was made of the stationarity of $\mathbf{V}_{\mathbf{n}}(t)$ as well as of the normalization of $h(t)$ to unit area. Simplification of (30) takes some more steps and the additional assumption of the Gaussian nature of $\mathbf{V}_{\mathbf{n}}(t)$, as in this case the moment theorem of Gaussian processes (see, e.g., [5]) can be employed. After some lengthy but straightforward manipulations, we finally arrive at

$$
\begin{aligned}
\sigma_{\mathbf{i}}^{2}(t)= & \sigma_{\mathbf{i}, \text { signal shot }}^{2}(t)+\sigma_{\mathbf{i}, \text { ASE shot }}^{2}(t) \\
& +\sigma_{\mathbf{i}, \text { ASE-ASE }}^{2}(t)+\sigma_{\mathbf{i}, \mathrm{s} \text {-ASE }}^{2}(t)
\end{aligned}
$$

with the signal shot noise

$$
\sigma_{\mathbf{i}, \text { signal shot }}^{2}(t)=S e\left(p * h^{2}\right)(t)
$$

the ASE shot noise

$$
\sigma_{\mathbf{i}, A S E}^{2} \text { shot }(t)=2 S e P_{n} B_{h}
$$

the signal-ASE beat noise

$$
\begin{aligned}
\sigma_{\mathbf{i}, s-\mathrm{ASE}}^{2}(t)=2 S^{2} \Re & \left\{\int_{-\infty}^{\infty} \int_{s} V_{s}(\tau) V_{s}^{\star}(\tilde{\tau})\left\langle\mathbf{V}_{\mathbf{n}}(\tau) \mathbf{V}_{\mathbf{n}}{ }^{\star}(\tilde{\tau})\right\rangle\right. \\
& \times h(t-\tau) h(t-\tilde{\tau}) d \tau d \tilde{\tau}\}
\end{aligned}
$$

and the ASE-ASE beat noise

$$
\begin{aligned}
\sigma_{\mathbf{i}, \mathrm{ASE}-\mathrm{ASE}}^{2}(t)= & S^{2} \int_{-\infty}^{\infty}\left|\left\langle\mathbf{V}_{\mathbf{n}}(\tau) \mathbf{V}_{\mathbf{n}}{ }^{\star}(\tilde{\tau})\right\rangle\right|^{2} \\
& \times h(t-\tau) h(t-\tilde{\tau}) d \tau d \tilde{\tau} .
\end{aligned}
$$

The symbol * means complex conjugation and $\Re$ denotes the real part. Substituting $t-\tau=\xi$ and $t-\tilde{\tau}=\eta$ in (36), 
and taking note of the stationarity of $\mathbf{V}_{\mathbf{n}}(t)$, the ASE-ASEbeat noise is immediately identified to be stationary. Further simplifications of (35) can only be obtained if the bandwidth $B_{O}$ of the optical filter following the optical amplifier is much larger than both the electrical bandwidth $B_{h}$ and the signal bandwidth (which is true for most technically relevant cases); then, the ASE field's autocorrelation function $\left\langle\mathbf{V}_{\mathbf{n}}(\tau) \mathbf{V}_{\mathbf{n}}{ }^{\star}(\tilde{\tau})\right\rangle$ can be approximated by the Dirac $\delta$-functional $\left(P_{n} / B_{0}\right) \delta(\tau-$ $\tilde{\tau})$ within the integral in (35) and we arrive at

$$
\sigma_{\mathbf{i}, s-\mathrm{ASE}}^{2}(t)=2 \frac{P_{n}}{B_{O}} S^{2}\left(p * h^{2}\right)(t)
$$

for the signal-ASE beat noise.

We thus conclude from (37) that the $s$-ASE beat noise variance is of the same form as the ordinary shot noise variance (2), and from (36) that the ASE-ASE beat noise term is stationary.

\section{ACKNOWLEDGMENT}

The authors are thankful for many fruitful discussions with W. R. Leeb. A discussion on the influence of intersymbol interference with $\mathrm{H}$. Weinrichter is also acknowledged.

\section{REFERENCES}

[1] S. D. Personick, "Receiver design for digital fiber optic communication systems I," Bell Syst. Tech. J., vol. 52, pp. 843-874, 1973.

[2] L. Boivin, M. C. Nuss, J. Shah, D. A. B. Miller, and H. A. Haus, "Receiver sensitivity improvement by impulsive coding," IEEE Photon. Technol. Lett., vol. 9, pp. 684-686, 1997.

[3] S. Tanikoshi, K. Ide, T. Onodera, Y. Arimoto, and K. Araki, "High sensitivity $10 \mathrm{~Gb} / \mathrm{s}$ optical receiver for space communications," in Proc. 17th AIAA Int. Commun. Satellite Syst. Conf., 1998, pp. 178-183.

[4] T. Matsuda, A. Naka, and S. Saito, "Comparison between NRZ and RZ signal formats for in-line amplifier transmission in the zero-dispersion regime," J. Lightwave Technol., vol. 16, pp. 340-348, 1998.

[5] L. Mandel and E. Wolf, Optical Coherence and Quantum Optics. Cambridge, U.K.: Cambridge University Press, 1995.
[6] B. E. A. Saleh and M. C. Teich, Fundamentals of Photonics. New York: Wiley, 1991

[7] A. A. Abidi, "On the choice of optimum FET size in wide-band transimpedance amplifiers," J. Lightwave Technol., vol. 6, pp. 64-66, 1988.

[8] S. D. Personick, "Receiver design for optical systems," in Proc. IEEE, vol. 65 , pp. 1670-1678, 1977.

[9] R. M. Gagliardi and S. Karp, Optical Communications. New York: Wiley, 1995.

[10] P. J. Winzer, "Shot-noise formula for time-varying photon rates: A general derivation," J. Opt. Soc. Amer. B, vol. 14, pp. 2424-2429, 1997.

[11] T. Li and M. C. Teich, "Photon point process for travelling-wave laser amplifiers," J. Lightwave Technol., vol. 29, pp. 2568-2578, 1993.

[12] P. J. Winzer, "Linking equations between photon statistics and photocurrent statistics for time-varying stochastic photon rates," accepted for publication in J. Europ. Opt. Soc., vol. 10, 1998.

[13] R. Ramaswami and P. H. Humblet, "Amplifier induced crosstalk in multichannel optical networks," J. Lightwave Technol., vol. 8, pp. 1882-1896, 1990.

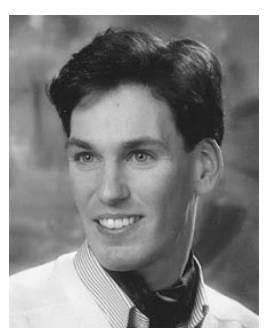

Peter J. Winzer was born in Vienna, Austria, in January 1973. He received the Dipl.-Ing. degree in electrical engineering from the Vienna University of Technology, Austria, in 1996. His doctoral thesis will deal with noise in Doppler wind lidar systems.

Since then he has been working on projects related to both space-borne wind lidar systems and optical intersatellite communications.

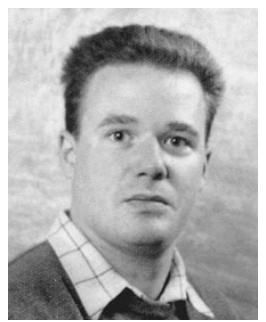

András Kalmár was born in Budapest, Hungary, in December 1971. He received the Dipl.-Ing. degree in electrical engineering from the Vienna University of Technology, Austria, in 1995. Since 1995, he has been working toward the Ph.D. degree on optical phased array antennas for space communications.

$\mathrm{He}$ is also involved in a project on optical intersatellite communications. 